\title{
Dermatitis aerotrasportada de origen laboral en la industria química, farmacéutica y sector sanitario
}

\author{
Airborne contact dermatitis in chemical and pharmaceutical industry and \\ healthcare sectors
}

\author{
Jaureguizar Cervera E. 1,2; Pérez Perdomo M. 1,2; Reinoso Barbero, L. ${ }^{3}$
}

1. Departamento Medicina del Trabajo. Hospital Universitario de la Princesa. Madrid. España.

2. Unidad Docente de Medicina del Tabajo de la Comunidad de Madrid. Hospital Universitario de la Princesa. Madrid. España.

3. Médico del Trabajo del Banco Popular. Madrid. España.

Recibido: 23-01-14

Aceptado: 17-07-14

\section{Correspondencia}

Enrique Jaureguizar Cervera

Correo electrónico: dr.jaureguizar@gmail.com

Resumen

Introducción: La dermatitis de contacto aerotransportada (DCAT), es una forma de dermatitis profesional que por su manifestación clínica, localización y forma de contacto presentan característica propias, este hecho junto con la relevancia de la dermatosis dentro de la morbilidad profesional justifica la necesidad de sintetizar la evidencia científica existente mediante una revisión sistemática.

Objetivos: Identificar la evidencia científica sobre la DCAT, la existencia de un consenso sobre criterios diagnósticos, las medidas de prevención y la identificación de población especialmente sensible.

Material y métodos: Revisión sistemática de la producción científica publicada entre 2006 y 20014 sobre DCAT, se realizó una búsqueda sistemática mediante términos DeCS, MeSH, en diversas bases de datos y otros recursos informáticos (MEDLINE, COCHRANE, SCOPUS, SCIELO, OVID, BNCS, OSH, UPDATE). La evidencia se evaluó con los criterios SIGN.

Resultados: Tras el proceso de selección el resultado final fue de $\mathbf{1 5}$ artículos a analizar del total recuperado de 504. Los tipos de diseño: 1 Ensayo Clínico no aleatorizado sin grupo control y 14 Series de Casos, de los cuales 7 usaron grupo control. Las sustancias químicas que produjeron DCAT no publicadas previamente fueron: TFA, fibras PP y PE, Dibidrocloruro 2 2'-azobis (2-metilpropanamida) y HBTU ${ }^{1}$; y los fármacos: Tilosina, Pristinamicina, Carbocromeno y Famotidina.

Discusión y conclusiones: La limitación de los resultados es el bajo nivel de evidencia científica de los artículos analizados, lo que compromete su validez externa. Con respecto a las revisiones de Santos $\mathrm{R}$, y Grosens A (2001 al 2006) encuentran 9 casos nuevos de DCAT y Swinnen I, y Grossens A (2007-2011) describen 14 casos. En nuestra revisión (2006-2014) hay 6 nuevos casos, lo que reafirma un aumento en el interés del estudio y la trascendencia de esta patología. 
No se puede demostrar ninguna población especialmente sensible, se puede concluir que la DCAT está cobrando importancia, y que se debe mejorar la producción científica con diseños que generen un mayor nivel de evidencia que permitan tomar las medidas preventivas adecuadas.

Med Segur Trab (Internet) 2014; 60 (237) 742-755

Palabras clave: Dermatitis de contacto; aerotransportada; alérgica; irritante; salud laboral; industria química; industria farmacéutica; personal sanitario; prevención.

1: TFA: Ácido TriFluoroAcético; Fibras PP: Polipropileno y PE: Polietileno; HBTU: 2-[(1H-Benzotriazol-1-il)-1,13,3 Tetrametilaminio Hexafluorofosfato].

Abstract

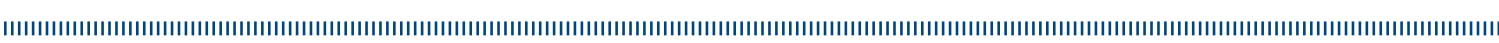

Introduction: Due to its location, clinical signs and form of contact, the airborne contact dermatitis (ABCD) is a unique type of occupational dermatitis. It results unquestionable the need of synthetising the scientific evidence through a systematic review taking into account the dermathoses relevance in the occupational morbidity.

Objectives: To identify the scientific evidence for ABCD, the existence of a consensus on diagnostic criteria, on preventive measures and on the identification of especially sensitive population.

Material and metbods: A systematic review of the scientific literature published between 2006 and 2014 about ABCD. A systematic search was performed using DeCS, MeSH, in various databases and other computer resources (MEDLINE, COCHRANE, SCOPUS, SCIELO, OVID, BNCS, OSH, UPDATE). The evidence was evaluated following a SIGN criteria.

Results: 15 articles were selected to be analyzed after a selecting process of 504. Types of design: 1-Non-randomized clinical trial without control group and 14 Case Series, 7 of which were using a control group. The ABCD chemicals not previously published: TFA, PP/PE fibers, Ethyl 2 2'-azobis Dibidroclorure (2- methyl propanamide) and HBTU ${ }^{1}$, and the drugs: Tylosin, Pristinamycin, Carbocromen and Famotidine.

Discussion and conclusions: The limitation of the results reveals the low scientific evidence levels on the analyzed articles, compromising his external validity. Regarding the reviews of Santos R, and Grossens A. (2001-2006), 9 new cases of ABCD were found and Swinnen I, and Grossens A. (2007-2011) described 14 cases. In our review (2006-2014) there are 6 new cases, confirming an increasing interest in this study and the gravity of this disease.

As it is impossible to demonstrate a particularly sensitive population, it can be concluded that the ABCD is gaining importance. The scientific production should be improved with designs which generate a higher level of evidence allowing to take preventive measures.

Med Segur Trab (Internet) 2014; 60 (237) 742-755

Keywords: Contact dermatitis; airborne; allergic; irritating; occupational health; chemistry industry; pharmaceutical industry; bealthcare workers; prevention.

1: TFA: TriFluoro acetic acid; PP Fibers: Polypropylene and PE: Polyethylene; HBTU 2 - [(1H-benzotriazol-1-yl) $-1,13,3$ Tetramethylammonium hydroxid, Hexafluorophosphate]. 


\section{INTRODUCCIÓN}

La dermatitis de contacto (DC) profesional engloba al conjunto de patologías cutáneas presentes en el mundo laboral. Su frecuencia se haya algo subestimada debido a la gran diversidad de lesiones que producen y su capacidad de afectar a todo el tegumento, solapándose de este modo con otras dermatitis.

La DC se puede definir como una reacción inflamatoria aguda o crónica de la piel al contacto directo con agentes nocivos, en concentración y período de tiempo suficiente para provocar una alteración con morfología claramente objetivable ${ }^{1,2}$. El daño que pueden originar dependerá de la capacidad de recuperación cutánea, que es variable de un individuo a otro ${ }^{3}$.

Las DC constituyen alrededor del 90\% del amplio espectro de las dermatosis profesionales, y se pueden dividir en dos formas: A) Dermatitis de contacto irritativa (DCI), que comprende el $80 \%$ de los casos, y B) Dermatitis de contacto alérgica (DCA) que es la responsable del $20 \%$ de los $\operatorname{casos}^{4}$.

La DCI es una reacción inflamatoria causada por la acción citotóxica directa del agente agresor a las células de la epidermis y la dermis, sin la producción de anticuerpos específicos, que produce una reacción cutánea localizada. La DCA, sin embargo, es una reacción inmunológica de hipersensibilidad retardada o tipo IV, mediada por células $\mathrm{T}$ y que requiere una sensibilización previa ${ }^{4,5}$, y que a su vez se van a dividir en: asimétrica.

- Agudas: aparece a las 24-48 h de la exposición y producen eccemas de localización

- Crónicas: aparecen por contacto repetido. Tienden a mostrar localizaciones simétricas con bordes imprecisos. Presentan una característica muy importante que es su tendencia a diseminarse a zonas distales.

Dentro de las dermatitis de contacto existen formas particulares como la dermatitis de contacto aerotransportada (DCAT), producidas por sustancias de carácter alérgico (DATA) o irritativo (DATI) que son liberadas a la atmósfera y luego se depositan sobre áreas de la piel ${ }^{6}$.

Agentes que pueden producir un cuadro de este tipo son gases y vapores, productos químicos contenidos en partículas sólidas o gotas y que, al contactar con la piel, al inhalarse o por ambos mecanismos, producen lesiones eccematosas monomorfas en cada paciente.

Las lesiones se localizan principalmente en la cara, donde la región palpebral superior es particularmente susceptible a los alérgenos transportados por el aire. Otras localizaciones típicas son: retroauricular, submentoniana, cuello, dorso de las manos ${ }^{7}$ También es frecuente que las lesiones aparezcan en los grandes pliegues y miembros inferiores, debido a que estas sustancias se depositan en la ropa y, junto con la retención del sudor, causan dermatitis en zonas ocluidas ${ }^{8}$.

Existen diversas clasificaciones de las DCAT en función de la sintomatología que producen: alérgica: maderas, plantas, plásticos, insecticidas, pesticidas; irritativa: gas mostaza $^{9}$, fibra de vidrio ${ }^{10}$, fibra de carbono ${ }^{11}$, oxido de etileno ${ }^{12} ;$ fototóxicas: psolarenos $^{13}$ y colofonia ${ }^{14}$; fotosensibles: tioureas, pesticidas ${ }^{15}$; Reacciones acneiformes: $\mathrm{PVC}^{16}$; urticaria de contacto: látex ${ }^{17}$, resina epoxi; Lesiones purpúricas: resina epoxi ${ }^{18}$; alteraciones de la pigmentación: maderas tropicales como Plathymenia foliosa ${ }^{19}$; otras: dermatitis exfoliativa, exantema fijo, erupciones liquenoides, dermatitis de contacto linfomatoidea, parestesia, reacciones pustulosas, telangiectasias, y eritema multiforme ${ }^{20}$.

La variabilidad de las manifestaciones clínicas va a depender del agente causal, sin olvidar que una misma sustancia puede producir distintas reacciones.

En 1939, la Asociación Médica Americana define la dermatitis profesional como «una afectación de la piel en la que puede encontrarse que el trabajo es su causa fundamental o un factor que contribuye a ella». Esta definición fue modificada en el X Congreso Latinoamericano de Dermatología de 1983 como «toda alteración de la piel, mucosas y anexos, directa o indirectamente causada, condicionada, mantenida o agravada por todo aquello que sea utilizado en la actividad profesional o exista en el ambiente de trabajo» ${ }^{21}$. 
La DCAT está declarada como enfermedad profesional ${ }^{22}$ según el Real Decreto 1299/2006, de 10 de noviembre. Pertenece al Grupo 5 - AGENTE A - SUBAGENTE 01 de enfermedades profesionales, que engloba a todas aquellas dermatitis causadas por sustancias de bajo peso molecular por debajo de los 1000 daltons, tales como metales y sus sales, polvos de maderas, productos farmacéuticos, sustancias químico plásticas, aditivos, disolventes, conservantes, catalizadores, perfumes, adhesivos, acrilatos, resinas de bajo peso molecular, formaldehídos y derivados, etc.

Por esta razón, hoy día para clasificar una dermatitis como enfermedad ocupacional se tiene que cumplir una serie de hechos ${ }^{7,23}$ : Historia clínica laboral concordante, la localización de las lesiones se deben corresponder en zonas que se relacionen con la forma y tipo de trabajo, pruebas de contacto positivas, estudios en controles voluntarios con concentraciones no irritantes cuando se desconozcan las concentraciones adecuadas, mejoría cuando no se trabaja o cuando se evite el contacto con el alérgeno implicado.

La DC constituye una de las enfermedades profesionales más frecuentes en los países industrializados siendo, en algunos de ellos, las primeras enfermedades profesionales notificadas y más del $30 \%$ de las que requieren compensación económica ${ }^{7,24}$. Informes de proyectos de vigilancia de enfermedades cutáneas, Epiderm y Opra, sugieren una tasa de incidencia de 13 por 100.000 por año, y una prevalencia de 15 por 10.000 trabajadores $^{25}$.

El riesgo de desarrollar dermatitis de origen ocupacional varía en relación con la profesión. Entre el alto riesgo están los peluqueros (tasa anual de 120/100.000), operarios de maquinaria (tasa anual 56/100.000), trabajadores de industria química y petroleras (tasa anual 45/100.000) y ensambladores (tasa anual 35/100.000) ${ }^{26}$.

El presente trabajo está dirigido a identificar, mediante una revisión sistemática, la evidencia existente en relación con el manejo clínico y preventivo de las DCAT a partir de los artículos publicados entre enero de 2006 a enero de 2014, en el ámbito de la industria química, farmacéutica, y trabajadores del sector sanitario, la identificación de la población especialmente sensible de sufrir DCAT y la existencia de un consenso con respecto a los criterios de diagnóstico y las medidas preventivas a adoptar.

\section{MATERIAL Y MÉTODOS}

Se realizó una búsqueda sistemática de artículos científicos publicados entre los años 2006-2014, siendo la última fecha consultada enero de 2014. Se incluyen tres estudios publicados con anterioridad por su relevancia para nuestro trabajo.

La estrategia de búsqueda incluyó los términos DeCS y MeSH (Tabla I), con los que se construyeron las ecuaciones de búsqueda, posteriormente aplicadas sobre las bases de datos que figuran en la Tabla II.

Tabla I. Términos DeCS: «Descriptores en Ciencias de la Salud» empleados en las ecuaciones de búsqueda

\begin{tabular}{l}
\hline \multicolumn{1}{c}{ Descriptores } \\
\hline - Chemical Compounds: Compuesto químico. \\
- Dermatitis: Dermatitis. \\
- Dermatitis occupational: Dermatitis laboral. \\
- Particulate Matter. Airborne particulates: Aerotransportada. \\
- Dermatitis, contact: Dermatitis de contacto. \\
- Disease, occupational: Enfermedad laboral. \\
- Drug industry: Industria farmacéutica. \\
- Chemical industry: Industria química. \\
\hline
\end{tabular}


Tabla II. Bases de Datos y ecuaciones de búsqueda empleados para la identificación de publicaciones

\begin{tabular}{|c|c|}
\hline Base de Datos & Ecuaciones de búsqueda \\
\hline MEDLINE, via PubMed & 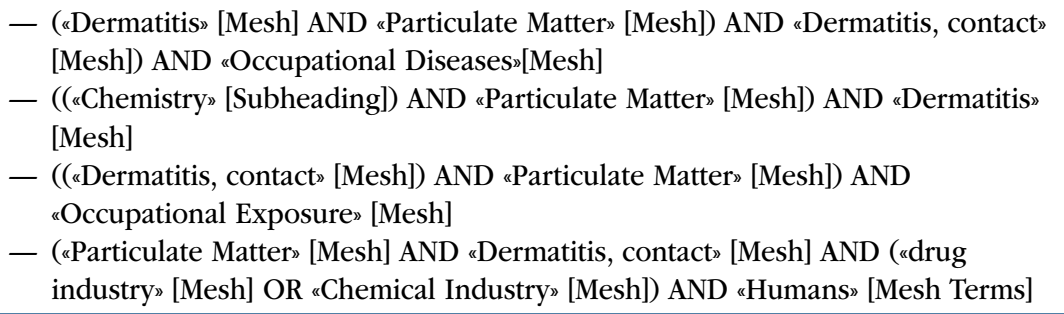 \\
\hline COCHRANE PLUS & "Particulate Matter» AND «contact dermatitis» \\
\hline EMBASE & "Particulate Matter» AND "contact dermatitis» \\
\hline SCOPUS & "Particulate Matter» AND «contact dermatitis» \\
\hline SciELO España & Dermatitis de contacto, "contact dermatitis" \\
\hline IBECS & "Particulate Matter» AND "contact dermatitis» \\
\hline WOK & "Particulate Matter" AND "contact dermatitis" \\
\hline WOS & "Particulate Matter» AND "contact dermatitis» \\
\hline OvidSP & $\begin{aligned} \text { - Dermatitis occupational AND chemical industry }\{\text { Incluyendo términos relacionados }\} \\
\text { - Dermatitis occupational AND chemical industry AND airborne particulates } \\
\text { \{Incluyendo términos relacionados }\}\end{aligned}$ \\
\hline
\end{tabular}

MEDLINE (Medlars Online International Literature), Cochrane library plus (Biblioteca Cochrane plus en español), EMBASE (Excepta Medical data base), IBECS (Índice Bibliográfico Español en Ciencias de la Salud), WOK (Web of Knowledge), WOS (Web of Science).

También se realizó una búsqueda en revistas especializadas en dermatología: Contact dermatitis, Clinical and Experimental Dermatology, y Dermatitis.

A los artículos recuperados tras la aplicación de las estrategias de búsqueda se les aplicaron unos criterios de inclusión y exclusión que se exponen en las tablas III y IV.

Tabla III. Criterios de inclusión

\begin{tabular}{ll}
\hline \multicolumn{1}{c}{ Variables } & \multicolumn{1}{c}{ Criterios de inclusión } \\
\hline Ámbito temático & $\begin{array}{l}\text { Relacionados con sustancias aerotransportadas y dermatosis profesionales en la } \\
\text { industria química-farmacéutica y en trabajadores sanitarios. }\end{array}$ \\
\hline Tipo de diseño & $\begin{array}{l}\text { Estudios Experimentales. } \\
\text { Estudios analíticos o longitudinales. } \\
\end{array}$ \\
Estudios descriptivos o transversales. \\
\hline Población & Trabajadores de la industria química, farmacéutica y profesional sanitario. \\
\hline Artículos publicados & Fecha: Posteriores a 2006 inclusive. \\
\hline Idiomas & Español, inglés, francés, portugués. \\
\hline
\end{tabular}

Tabla IV. Criterios de exclusión

\begin{tabular}{ll}
\hline \multicolumn{1}{c}{ Variables } & \multicolumn{1}{c}{ Criterios de exclusión } \\
\hline Criterios de inclusión & No cumplir los criterios anteriores. \\
\hline Tipo de diseño & Editoriales, cartas al director, opiniones de expertos. \\
\hline Tipo de estudio & $\begin{array}{l}\text { Estudios relacionados con plantas, agentes biológicos vivos, metales. } \\
\text { Ámbito no laboral: contaminación ambiental, domicilio, infancia. }\end{array}$ \\
\hline Duplicados & En distintas bases de datos/autor. \\
\hline Estudios no originales & Estudios de revisión sin metodología sistemática.
\end{tabular}


Tras la aplicación de los criterios de selección, los artículos sobre los que existía alguna controversias en relación con la pertinencia de su inclusión o no en la revisión, se re-evaluaron de forma conjunta llegando a un consenso sobre la pertinencia de su inclusión en la revisión sistemática.

Para determinar el nivel de evidencia científica y grados de recomendación de cada artículo, se evaluó con los criterios SIGN de la «Scottish Intercollegiate Guidelines Network» 27.

Posteriormente se procedió a la recuperación de los artículos a texto completo en la Escuela Nacional de Medicina del trabajo y la Biblioteca Nacional de Ciencias de la SaludInstituto de Salud Carlos III.

\section{Resultados}

La Tabla V refleja el número total de artículos recuperados en cada una de las bases de datos consultadas tras aplicar las distintas ecuaciones de búsqueda. Se obtuvo un total de 504 artículos, así como el número de artículos seleccionados finalmente para su revisión de acuerdo al proceso de selección realizado (Figura 1).

Tabla V. Número de artículos recuperados y seleccionados

\begin{tabular}{ccc}
\hline Base de datos & $\begin{array}{c}\text { Artículos } \\
\text { recuperados }\end{array}$ & $\begin{array}{c}\text { Artículos } \\
\text { seleccionados }\end{array}$ \\
\hline Google Accademics & 64 & 0 \\
Pub Med & $\mathbf{3 8 6}$ & $\mathbf{1 5}$ \\
COCHRANE & 5 & 0 \\
SCOPUS & 24 & 0 \\
SCIELO & 4 & 0 \\
OVID & 12 & 0 \\
BNCS & 8 & 0 \\
OSH UPDATE & 1 & 0 \\
Total de artículos & $\mathbf{5 0 4}$ & $\mathbf{1 5}$ \\
\hline
\end{tabular}

Tras el proceso de selección el resultado final fue de $\mathbf{1 5}$ artículos a analizar. Desde el punto de vista del diseño metodológico el tipo de estudios incluidos fueron: 1 Ensayo Clínico no aleatorizado y 14 Series de Casos, de los cuales 7 usaron grupo control.

Figura 1. Resultados de la búsqueda

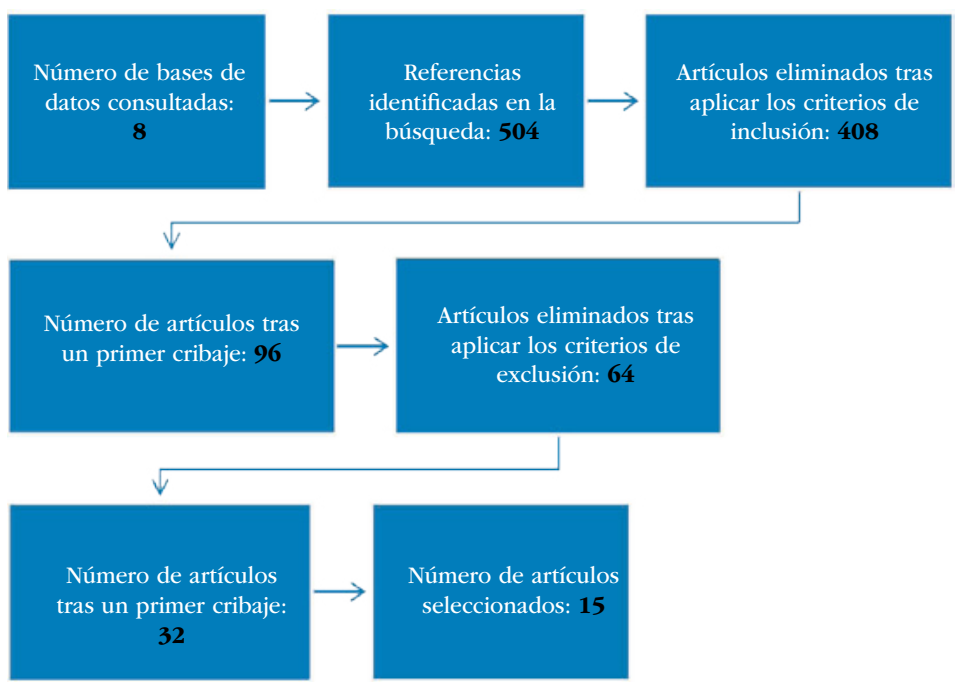


Las series clínicas con un mayor número de casos descritos se detallan a continuación, siendo la última publicación de 2013 donde Swinnen I. ${ }^{28}$ analiza 4 casos de mujeres que trabajan aplastando comprimidos de varios tipos de medicamentos y desarrollan cuadros de dermatitis aerotransportada severa. Los autores buscan establecer qué alérgenos son culpables de este cuadro. Para ello realizan pruebas epicutáneas estándar de la serie europea diluidos a diferentes concentraciones, incluyendo también los fármacos que los trabajadores usaban habitualmente en su puesto de trabajo. Los resultados de la lectura se levantan a las 48h (D2) y 96 h (D4) siguiendo el protocolo de la International Contact Dermatitis Research Group (ICDRG). Se observa que en los 4 casos existe respuesta positiva para las benzodiacepinas en D2 y D4, y dentro de este grupo, el que mayor respuesta alérgena presenta es el tetrazepam. Otros fármacos estudiados fueron antidepresivos, hipnóticos, betabloqueantes, IECAS e IBP. En estos casos los resultados difieren entre los cuatro trabajadores, dentro de éstos el hidrocloruro de trazodona fue el que más respuesta positiva dio ( 2 de 4 casos tanto en D2 y D4). Los autores intentan determinar si existe sensibilización cruzada entre las benzodiacepinas, concluyendo que probablemente las reacciones de sensibilidad observadas no sean debidas a una reacción cruzada, sino a una diferencia a nivel molecular, que hace que el tetrazepam tenga mayor capacidad de producir reacciones de sensibilidad respecto al resto.

Malaiyandi V. ${ }^{29}$ (2012) estudia a dos mujeres auxiliares de farmacia que se encargaban de la granulación de comprimidos de tilosina, con el objetivo de determinar si existe reacción de sensibilización cruzada entre los diferentes macrólidos. Para ello realizan pruebas epicutaneas según la North American Standard con diferentes fármacos del grupo de los macrólidos a distintas concentraciones. Observan que las respuestas más alergénicas corresponden a la tilosina, positiva en los dos casos, con D2-D5 ++ en la primera y D5 + en la segunda, y la clindamicina al 1\%, D5 + en la primera trabajadora. Comparan los resultados con un grupo control voluntario de 5 trabajadores observando respuesta negativa en todos, concluyendo que no existe tal reacción de sensibilidad cruzada.

Braun-Falco $\mathbf{M}^{30}$ (2008) realiza un estudio en 4 trabajadores (3 varones y 1 mujer) que habían desarrollado DCAT secundaria a Kathon CG (metilisotiazolina (MI) y metilcloroisotiazolina (MCI)), conservante antibacteriano y antifúngico utilizado en cosméticos, productos industriales y productos domésticos. No existe ni aleatorización ni grupo control. Se somete a los trabajadores a una exposición directa durante 2 horas a $28^{\circ} \mathrm{C}$, en una habitación de $16 \mathrm{~m}^{2}$ recién pintada con Alpina Sensan (conservante libre de biocida) con el objetivo de determinar una menor capacidad irritativa de este producto respecto al Kathon. Para ello miden $1 \mathrm{~h}$ antes y después la TEWL (agua transdérmica perdida por exposición) y el flujo sanguíneo en cara volar de antebrazos usando Tewameter para TEWL y Láser Doppler para flujo dermosanguíneo. Los resultados obtenidos demostraron la ausencia de síntomas cutáneos tras la exposición, por lo que los autores afirman el beneficio de usar pinturas libres de conservantes en personal con historia conocida de sensibilización a las isotiazolinas.

Jiska A. ${ }^{31}$ (2005) estudia a 5 mujeres trabajadoras en diferentes sectores de la UCI ( 2 fisioterapeutas, 2 enfermeras y 1 auxiliar) que desarrollan una clínica compatible con DCAT. Este estudio pretende mostrar la capacidad alergénica de las fibras de polipropileno (PP) y polietileno (PE) utilizadas en los filtros del aire acondicionado, y que en determinadas circunstancias ambientales, como en las salas de UCI, donde se trabaja a baja humedad y altas presiones en los filtros del aire acondicionado, pueden producir DCAT por liberación de dichas partículas. Es una DCAT principalmente irritativa, ya que en todos los casos estudiados, lo síntomas desaparecían con tratamiento tópico. Por ello enfatizan en el control y el correcto mantenimiento de las salidas de filtro de aire acondicionado. La limitación que encontraron los autores a la hora de realizar el estudio fue la falta de información por parte del fabricante acerca del diámetro, la longitud y el revestimiento de las fibras que componen el aire acondicionado. 
Conde-Salazar L. ${ }^{32}$ (2004) estudia 18 trabajadores de la industria químicofarmacéutica que intervienen en la elaboración de los medicamentos entre los años 19842002. Estos casos presentaban lesiones cutáneas compatibles con DCAT, por lo que se realizaron pruebas epicutáneas estándar del GEIDC junto a productos propios con los que contactaban cada uno de los trabajadores. La lectura se realizó en D2 y D4, encontrándose que los agentes más sensibilizantes eran el carbocromeno y la famotidina (+++ en 6 de los 18 pacientes estudiados, 3 para cada fármaco), frente a grupo control de 25 voluntarios. Estos autores concluyen en la importancia de realizar pruebas epicutáneas en aquellos trabajadores con sospecha de DCAT incluyendo todas las sustancias con las que los individuos podrían estas en contacto, tanto el principio activo como el producto final del mismo.

Dentro de los artículos que presentan casos aislados es el de Yeon Byun J. ${ }^{33}$ el más reciente (enero de 2014). Presenta un caso de DCAT en un trabajador técnico de laboratorio de la industria química que manipula diversos productos entre los que se encuentra el ácido trifluoroacetico (TFA), disolvente orgánico con múltiples usos. Se relaciona por primera vez esta sustancia con la posibilidad de aparición de DCAT, según los resultados de las pruebas epicutáneas que fueron positivas en el caso estudiado frente a los 5 controles sanos negativos, la localización de las lesiones y la ausencia de las mismas al retirarle del puesto de trabajo, sugieren su relación laboral.

Geier J. ${ }^{34}$ (2013) estudian el caso de una enfermera que desarrolla DCAT por didecildimetilamonio (DDAC), químico usado como desinfectante, agente tensoactivo, suavizante de tela, agente antiestático y espermicidas. Las pruebas epicutáneas dieron resultado positivo a concentración de $0,1 \%$ y negativo a $0,01 \%$. Se comparó con un control en el que las pruebas epicutáneas fueron negativas. Los autores recomiendan usar pruebas epicutáneas con dos concentraciones diferentes de DDAC al 0,1\% y al 0,01\% para no infravalorar resultados.

Oliphant T. ${ }^{35}$ (2011) analiza el caso de un trabajador de mantenimiento en una fábrica de polímeros que desarrolla DCAT por dibidrocloruro 2- $2^{-}$azobis (2-metilpropioamidina), toma como grupo de control a 20 trabajadores sanos voluntarios, los resultados de las pruebas epicutáneas fueron positivos en el trabajador expuesto y negativo en los controles. La ficha técnica del producto no indica que produjera irritación. Los autores argumentan las dificultades para el desarrollo del estudio derivadas de la diferente denominación que recibe el mismo compuesto químico, lo que generara incertidumbre en relación al compuesto estudiado.

Neumark M. ${ }^{36}$ (2011) presenta un caso de DCAT debido al pantoprazol en un técnico de maquinaria de industria farmacéutica. Los estudios realizados hasta la fecha muestran DC y DCAT en otros fármacos de la familia como omeprazol, benzimidazol, o lansoprazol, pero éste es el primer caso descrito de DCAT por pantoprazol. Los resultados positivos a las pruebas cutáneas en el caso frente a la negatividad en 10 controles, así como la localización de las lesiones y la remisión tras 2 meses de evitar la exposición, evidencian la sensibilización al pantoprazol.

Neumark M. ${ }^{37}$ (2009) expone un caso de DCAT con angioedema debido al simvastatina, carvedilol y zolpidem en un técnico de maquinaria de industria farmacéutica. La asociación de cuadros de DCAT por simvastatina es conocida. Sin embargo es la primera vez que aparecen asociados con la exposición a carvedilol, y zolpidem. Los resultados positivos a pruebas cutáneas en el caso frente a la negatividad en 10 controles, así como la localización de las lesiones y la su remisión tras 3 meses de evitar la exposición, evidencian la sensibilización a la simvastatina.

Corazza M. ${ }^{38}$ (2008) presenta un caso de DCAT debido a budesonida en una trabajadora que administra este fármaco. Los resultados evidencian la asociación entre DC y DCAT, con distintos grupos de corticoides, presentando además una sensibilización 
cruzada hasta en el $90 \%$ de los casos, como se demostró con la bydrocortisona-17butyrate a la que el caso no había tenido antecedentes de contacto.

Isaksson M. ${ }^{39}$ (2007) presenta un caso de DCAT debido a metil metacrilato (MMA) en una enfermera dental atópica (sensible a níquel, cobalto, myroxylon, pereirae, mezcla de perfuma, colofonia, epoxi, formaldehido, cuaternium-15 y budesonida). Se estudió si el cuadro de dermatitis era debido a una exposición laboral por vía areotransportada. Confirmándose esta relación por la positividad de las pruebas epicutáneas, de acuerdo con ICDRG en D3 y D7 a MMA, trietilenglycol dimethacryalto (TEGDMA), etilenglycol dimethacryalto (EGDMA), 2,2-bis(4-(2-Hidroxi-3-metacriloxipoxi)fenil)-propano (bisGMA), 2-hydroxyethyl methacrylate (2-HEMA) la respuesta fue más intensa para las dos últimas, y débilmente positivas para metacrilatos. Las tomas de muestras se realizaron mediante una bomba de aire con filtro de teflón XAD-2, obteniéndose un solo resultado positivo en el polvo de un escritorio de la clínica.

Pontén A. ${ }^{40}$ (2006) estudia un caso de DCAT debido a budesonida en una auxiliar de enfermería en la que se confirmó una dermatitis aerotransportada en base a las características de las lesiones dérmicas, su distribución, los resultados de las pruebas epicutáneas, y la desaparición de las mismas una vez que el trabajador dejaba de estar en contacto con dicho agente. La concentración del fármaco en el ambiente era extremadamente baja, con lo que se llegó a la conclusión de que la paciente era altamente sensible a la budesonida.

Blancas R. ${ }^{41}$ (2006) analiza un caso de DCAT por pristinamicina en un trabajador de industria farmacéutica que se encarga de la granulación y compresión de distintos medicamentos. Se realizaron pruebas epicutáneas de la serie estándar GEIDC con resultados negativos. Las pruebas cutáneas se repitieron con diferentes concentraciones, dando D2 y D4 ++, frente a un grupo de 10 controles voluntarios, el los que las pruebas dieron resultados negativos. Los autores concluyen que este efecto es debido a las propiedades sinérgicas que comparte con la virginiamicina (otro macrólido), ya que ambos medicamentos presentan factores sensibilizantes comunes (factor IIA de la virgniamicina y M de pristinamicina). Por ello recomiendan el uso de medidas de protección y control de la higiene industrial en aquellos trabajadores expuestos a sustancias con alto poder irritante.

Bousquet PJ. ${ }^{42}$ (2005) expone un caso de HBTU (O-(benzotriazol-1-il)- $N, N, N$; $N$ '-tetrametiluronio hexafluorofosfato, químico usado en síntesis protéica) en un trabajador de la industria química que manipulaba este producto, el diagnóstico de DCAT se basó en las característica de las lesiones dérmicas, la distribución, los resultados de la biopsia, así como los resultados de las pruebas epicutáneas, y la desaparición de las mismas una vez que el trabajador dejaba de estar en contacto con dicho agente.

La Tabla VI sintetiza los resultados obtenidos de los artículos más relevantes. 


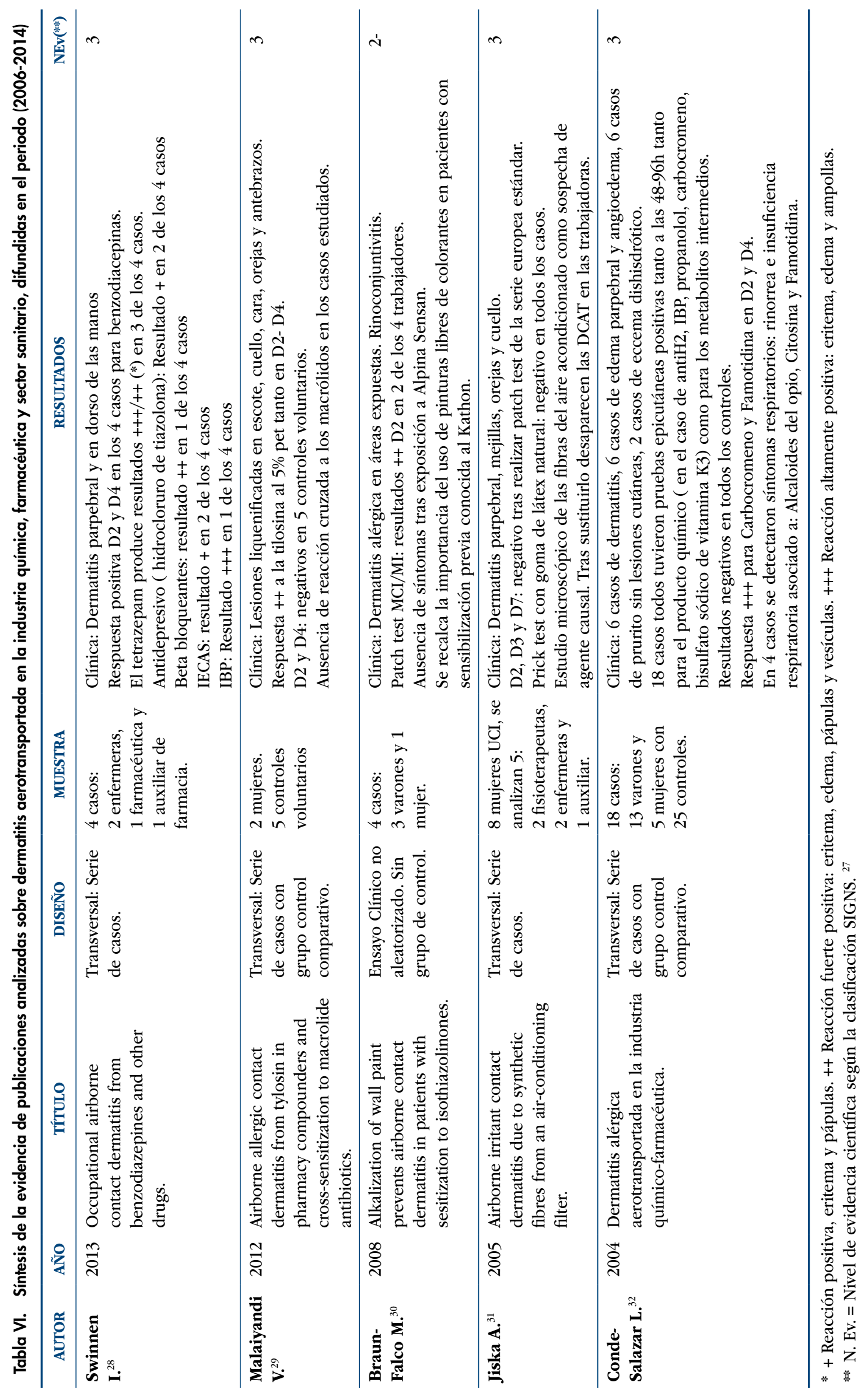




\section{DISCUSIÓN Y CONCLUSIONES}

La principal limitación encontrada ha sido el bajo nivel de evidencia científica de los artículos revisados. En general se tratan de series de casos y comunicaciones de casos puntuales, lo que implica una validez externa cuestionable debido a su reducido tamaño muestral.

La literatura científica recuperada, demuestra la asociación entre las DCAT y la presencia de sustancias irritantes en el lugar de trabajo ${ }^{43}$, en este caso, en la industria química, farmacéutica y en personal sanitario, reforzando el hecho de que nos encontramos ante una forma de presentación prevalente dentro de la patología dermatológica laboral ${ }^{32}$ y demostrando el interés y la relevancia del tema a nivel médico-laboral.

Swinnen I., tras la última revisión de DCAT $^{44}$, señala un aumento de los casos de dermatitis asociadas con la exposición a fármacos, encontrando 14 nuevos casos durante los 4 años de estudios, con respecto a una anterior revisión realizada, en la que sólo se encontraron $9 \operatorname{casos}^{45}$. En esta revisión, se han obtenido 6 nuevos casos de dermatitis por fármacos no clasificados como alergénicos aerotransportados en las revisiones realizadas entre los años 2006-2014, quedando así demostrado, un aumento en el interés del estudio y la trascendencia de las DCAT.

La budesonida y el tetrazepam son las sustancias que de forma mas frecuente han sido estudiadas en los últimos años como causantes de DCAT. Uno de los últimos estudios publicados acerca de las benzodiacepinas en profesionales sanitarios ${ }^{28}$, fue realizado con el propósito de determinar si esta familia de medicamentos presentaba reacción cruzada al usarlos conjuntamente. Aunque sus autores no pudieron concluir dicha reacción, sirvió para aumentar el convencimiento del efecto irritante del tetrazepam atribuible potencialmente a su diferente estructura química con respecto al resto de las benzodiacepinas. Sin embargo, los estudios analizados sobre la budesonida ${ }^{38}$, sí muestran la existencia de reacción cruzada con otro corticoide (hidrocortisona-17-burirato).

En esa misma revisión ${ }^{44}$ Swinnen et al. posicionan a los compuestos químicos como cuarta causa de DCAT, tras el grupo de fármacos, plantas y plásticos.

En esta revisión, se han encontrado 3 nuevos casos de DCAT por químicos comunicados en los últimos años ${ }^{33-35}$.

Dentro de las publicaciones más recientes cabe destacar el estudio realizado en 2008 por Braun-Falco M. sobre Kathon CG, éste agente es el principal irritante dermatológico de origen químico estudiado ${ }^{44,45}$, siendo este trabajo uno de los pocos estudios que relacionan la exposición a Kathon con la aparición de cuadros de dermatitis aerotransportada ${ }^{46}$.

También llama la atención los resultados del estudio más reciente publicado hasta la fecha (Enero 2014) acerca de la capacidad irritativa del TFA por vía aerotransportada ${ }^{33}$, un reactivo utilizado en síntesis orgánica, del cual no se había descrito en la literatura como agente causal de DCAT ${ }^{44-48}$.

A continuación, se detalla en la Tabla VII, los alérgenos encontrados en las últimas publicaciones analizadas, no identificados como agentes causantes de DCAT según la última revisión realizada entre los años 2007-2011 ${ }^{46,47}$.

Tabla VII. Nuevos alérgenos encontrados en las publicaciones entre 2006-2014

\begin{tabular}{ll}
\hline \multicolumn{1}{c}{ Fármacos } & \multicolumn{1}{c}{ Productos químicos } \\
\hline Tilosina & TFA (Ácido Trifluoroacético) \\
Carbocromeno & Dihidrocloruro 2 2'-azobis (2- metilpropanamida) \\
Famotidina & Fibras de polipropileno (PP) y polietileno (PE) \\
\hline
\end{tabular}


Los resultados de las publicaciones revisadas, junto a la información estadística disponible sobre notificación de enfermedad profesional en el período enerodiciembre 2013, ponen de manifiesto la importancia de las DCAT en el ámbito laboral, situándolas por su incidencia como la cuarta causa de enfermedad profesional con baja laboral.

Dentro de este grupo, las dermatosis producidas por sustancias de bajo peso molecular, entre las que se incluyen las DCAT, suponen la primera causa de baja ${ }^{49}$, si bien, con una menor duración de la baja laboral, con una media de 30,43 días por trabajador, afectando más al sexo femenino en los sectores analizados ${ }^{49}$.

Otro de los objetivos de esta revisión, es establecer, si en la literatura publicada existe evidencia científica sobre población especialmente sensible a desarrollar esta patología. Del total de 42 casos estudiados en las diferentes publicaciones, sólo 7 presentaban antecedentes de atopia o alergia, lo que puede hacer hipotetizar que este tipo de antecedente no tiene la influencia esperada en la aparición de síntomas de DCAT.

También se analizó la variabilidad o la armonización sobre los criterios de diagnóstico para este tipo de patología, observándose como punto común en los artículos analizados, la realización de pruebas epicutáneas o patch test con la batería estándar de alérgenos usados según diferentes países (GEIDAC, Europeas TRUE TEST, serie estándar Norteamericana, British Society for Cutaneus Allergy...) así como prick test o pruebas intradérmicas en los casos de sospecha de causa irritativa. La lectura de pruebas a las D2: 48 y D4: 96 horas, es también común en los casos presentados, aplicando los criterios de valoración $(+,++\mathrm{y}+++)$ recomendados por la ICDRG y en aquellos casos con resultado dudoso realizar una lectura tardía D5-D7.

Con respecto a las medidas de protección del trabajador, los artículos analizados no especifican si los trabajadores expuestos usaban equipos de protección, y en caso de que lo hicieran ${ }^{41}$, no aclaran si lo hacían de forma correcta. Por ello, se ha podido concluir que la mayor parte de los casos de DCAT en los trabajadores de la industria química, farmacéutica y sanitaria pueden ser debidos a una exposición directa al alérgeno, más que a una condición individual de susceptibilidad, teniendo en cuenta que la mayoría de los síntomas mejoran con un tratamiento tópico adecuado e incluso llegan a desaparecer cuando el trabajador deja de estar en contacto con el alérgeno ${ }^{42,33}$.

Por razones sociales, humanas y económicas los trabajadores expuestos a sustancias químicas dañinas deben estar protegidos para desarrollar sus actividades laborales.

Las medidas preventivas pueden reducir en gran parte el riesgo de las dermatitis profesionales. Las medidas de prevención individuales o colectivas son principalmente el uso de ropa protectora, evitar la exposición a materiales con potencial alergénico o tratar de sustituirlo por otro que carezca de esas propiedades, uso adecuado de agentes de limpieza y cremas de barrera, establecer condiciones de aseo personal que resulten fáciles y convenientes, automatizar el proceso si las condiciones de trabajo son peligrosas para el reducir el de tiempo de exposición a la sustancia peligrosa y supervisión, educación y buen mantenimiento y limpieza del sitio de trabajo, entre los que se incluyan sistemas de ventilación adecuados ${ }^{50,51}$.

Desde el punto de vista de la prevención, podemos concluir que el factor principal de riesgo es la exposición directa al alérgeno en el trabajo, viéndose asociadas principalmente sustancias de bajo peso molecular, que por sus características son más difíciles de identificar en el ambiente laboral y por lo tanto, pasan inadvertidas como causantes de la patología.

En función del estado de conocimiento actualmente existente sobre las DCAT, se puede concluir que la DCAT representa una forma de manifestación de la patología dermatológica de origen laboral, clínicamente bien descrita, pero sobre la que existe una escasa evidencia en relación a la historia natural de la enfermedad: etiología, vulnerabilidad, mecanismos de actuación, etc. Este hecho, unido al incremento en el número de casos 
informados en la literatura científica, la convierte en un tema de interés preferente en la investigación de la enfermedad profesional de naturaleza dermatológica.

\section{Conflicto de interés}

Los autores declaran no tener conflictos de interés.

\section{REFERENCIAS BIBLIOGRÁFICAS}

1. Lachapelle JM. Contact dermatitis. 2011; P1-9.

2. Tato R, Sanz J, del Campo M, Agulló A. Dermatitis de contacto en el medio laboral. Medicina del Trabajo 1999;(1): 11-17.

3. Diepgen T, Kanerva L. Occupational skin diseases. Eur J Dermatol. 2006;(3): 324-30.

4. Sasseville D. Occupational contact dermatitis. Allergy, Asthma and Clinical Immunology. 2008(2): 59-65.

5. Chew AL, Maibach HI. Occupational issues of irritant contact dermatitis. Int Arch Occup Environ Health. 2003;(5): 339-46.

6. Domingues JC, Gonçalo M, Gonçalo S. Dermatitis de contacto aerotransportadas. Med Cut. 1994; (22): $251-6$

7. Pere J. Editor. Manual de alergia cutánea. MRA 2011; P105-123.

8. Heras-Mendaza F, Conde-Salazar L. Wood related occupational contact dermatitis. Contact Dermatitis 2008; (Suppl. 1): 11.

9. Vena GA, Foti C, Grandolfo M, et al. Contact irritation as- sociated with airborne contact irritation from mustard gas. Contact Dermatitis 1994; (31): 130-1.

10. Verbeck SJ, Bruise-ban Unnick EMM, Malten KE. Itching in office workers from glass fibers. Contact Dermatitis 1981; (7): 354.

11. Eedy DJ. Carbon-fibre-induced airborne irritant contact dermatitis. Contact Dermatitis 1996; (35): 362-3.

12. Romaguera C, Vilaplana J. Airborne occupational contact dermatitis from ethylene oxide. Contact Dermatitis 1998; (39): 85.

13. Dooms-Goosens A, Deleu H. Airborne contact dermatitis: an update. Contact Dermatitis 1991; (25): $211-7$.

14. Lachapelle JM, Frimat $\mathrm{P}$, Tennsteddt $\mathrm{D}$, et al. Dermatoses aèroportees. Dermatologie Professionnelle et de l'Environnement.1992; 141-56.

15. Nakamura M, Arima Y, Nobuhara S, et al. Airborne photo-contact dermatitis due to the pesticides maneb and feni-trothion. Contact Dermatitis 1999; (40): 222-3.

16. Goh CL, Ho SF. An outbreak of acneiform eruption in a polyvinylchloride manufacturing factory. Dermatosen 1988; (36): 53-7.

17. Baur X, Ammon J, Chen Z, Beckmann U, Czuppon AB. Health risk in hospitals through airborne allergens for patients presensitised to latex. Lancet 1993; (342): 1148-9.

18. Downs AMR, Sansom J. Airborne contact dermatitis from epoxy resin in an immersion oil used for microscopy. Contact Dermatitis 1998; (39): 267.

19. Gawkrodger DJ. Patch testing in occupational dermatology. Occup Environ MEd. 2001; (12): 823-8.

20. Goossens A. Airborne dermatosis. Acta Dermatovenerol Croat 2006; (14): 153-55.

21. De la Fuente I, Bell B, Ibarz J. Estudio epidemiológico de las dermatosis profesionales en el hospital. Medicina del Trabajo. 1998; (6): 335-340.

22. Insht.net [Internet]. España. INSHT; 2006; [citado10 nov 2013]. Disponible en: http://www.insht.es/ InshtWeb/Contenidos/Normativa/TextosLegales/RD/2006/1299_2006/PDFs/ realdecreto12992006de10denoviembreporelqueseapruebael.pdf

23. Conde-Salazar L., Dermatología Laboral. Tratado de Medicina del Trabajo. 2005: 695.

24. Barchino Ortiz L, Cabrera Fernández E, Moreno Manzano G, Heras Mendaza F, Conde-Salazar Gómez L. Dermatosis profesionales en cuidadores de ancianos. Medicina y Seguridad del Trabajo 2007; (53): 35-46.

25. Gawkrodger DJ. Patch testing in occupational dermatology. Occup Environ Med. 2001; (12): 823-8.

26. Downs AMR, Sansom J. Airborne contact dermatitis from epoxy resin in an immersion oil used for microscopy. Contact Dermatitis 1998; (39): 267. 
27. Scottish Intercollegiate Guidelines Network. SIGN 50: 2001

28. Swinnen I, Ghys K, Kerre S, Constandt L, Goossens A.Occupational airborne contact dermatitis from benzodiazepines and other drugs. Contact Dermatitis. 2013 Nov 30.

29. Malaiyandi V, Houle MC, Skotnicki-Grant S., Airborne allergic contact dermatitis from tylosin in pharmacy compounders and cross-sensitization to macrolide antibiotics. Dermatitis. 2012 Sep-Oct; (5): 227-30.

30. Braun-Falco M, Knott E, Huss-Marp J, Ring J, Hofmann H. Alkalization of wall paint prevents airborne contact dermatitis in patients with sensitization to isothiazolinones, Contact Dermatitis. 2008; (2):129-31.

31. Patiwael JA, Wintzen M, Rustemeyer T, Bruynzeel DP. Airborne irritant contact dermatitis due to synthetic fibres from an air-conditioning filter. Contact Dermatitis. 2005 Mar; (3): 126-9.

32. Luis Conde-Salazar, Elena Vargasa, Ruud Valksa. Dermatitis alérgica aerotransportada en la industria químico-farmacéutica. Actas Dermosifiliogr. 2004; (95): 429-35.

33. Byun JY, Woo JY, Choi YW, Choi HY. Occupational airborne contact dermatitis caused by trifluoroacetic acid in an organic chemistry laboratory. Contact Dermatitis. 2014; (1): 63-4.

34. Geier J, Lessmann H, Krautheim A, Fuchs T. Airborne allergic contact dermatitis caused by didecyldimethylammonium chloride in a geriatric nurse. Contact Dermatitis. 2013; (2): 123-5.

35. Oliphant T, Wilkinson M, Wright A., Allergic contact dermatitis caused by 2,2-azobis(2methylpropionamidine) dihydrochloride used in the manufacture of acrylic polymer, Contact Dermatitis. 2011; (6): 356-7.

36. Neumark M, Ingber A, Levin M, Slodownik D., Occupational airborne contact dermatitis caused by pantoprazole. Contact Dermatitis. 2011; (1): 60-1.

37. Neumark M, Moshe S, Ingber A, Slodownik D. Occupational airborne contact dermatitis to simvastatin, carvedilol, and zolpidem. Contact Dermatitis. 2009; (1): 51-2.

38. Corazza M, Baldo F, Osti F, Virgili A. Airborne allergic contact dermatitis due to budesonide from profesional exposure. Contact Dermatitis. 2008; (59): 318-9.

39. Isaksson M, Zimerson E, Svedman C., Occupational airborne allergic contact dermatitis from methacrylates in a dental nurse. Contact Dermatitis. 2007 Dec; 57(6): 371-5.

40. Pontén A. Airborne occupational contact dermatitis caused by extremely low concentrations of budesonide. Contact Dermatitis. 2006 Aug; (2): 121-4.

41. Blancas-Espinosa R, Conde-Salazar L, Pérez-Hortet C. Occupational airborne contact dermatitis from pristinamycin. Contact Derrmatitis. 2006 Jan; 54(1): 63-5.

42. Bousquet PJ, Guillot B, Guilhou JJ, Raison-Peyron N. Occupational airborne allergic contact dermatitis due to HBTU. Contact Dermatitis. 2005; (1): 53-4.

43. Huygens S, Goossens A. An update on airborne contact dermatitis. Contact Dermatitis. 2001; (1): 1-6.

44. Schubert H. Airborne contact dermatitis due to methylchloro and methylisothiazolinone (MCI/MI). Contact Dermatitis 1997; 36: 274.

45. Ana Rita Rodrigues Barata, Jesús Rodríguez Espinosa, Felipe Heras Mendaza, Luis Conde-Salazar Gomèz. Kathon CG and Occupational Dermatology. An update. Med Segur Trab (Internet) 2012; 58 (228) $237-245$.

46. Swinnen I, Goossens A. An update on airborne contact dermatitis: 2007-2011. Contact Dermatitis. 2013; (4): $232-8$.

47. Santos R, Goossens A. An update on airborne contact dermatitis: 2001-2006. Contact Dermatitis. 2007; (6): 353-60

48. Eidman, KF, Nichols, L. Paquette. ed. Encyclopedia of Reagents for Organic Synthesis. 2004.

49. Sanjeev H, Dipankar D, Rahul M. Current perspectives in etiopathogenesis and management. Indian J Dermatol. 2011; (6): 700-706.

50. Seg-social.net [Internet]. España: CEPROSS-8. 2013. [citado 25 Enero 2013] Disponible en: http://www.segsocial.es/Internet_1/Estadistica/Est/Observatorio_de_las_Enfermedades_Profesionales/cepross2k11/ Partes_cerrados/Tablas/index.htm

51. Meza B. Dermatosis profesionales. Dermatología Peruana. 2006; (1): 67-68.

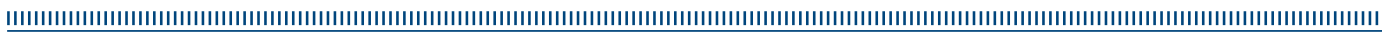

